\title{
Wide diversity of Crenarchaeota
}

SIR - The traditional perception of the diversity of the bacteria-like Archaea based on cultivation studies has been that the kingdom Euryarchaeota is a physiologically variable group including halophiles, thermophiles and methanogens, whereas the kingdom Crenarchaeota is more homogenous, consisting exclusively of sulphurdependent, extreme thermophiles ${ }^{1}$. This has led to the notion that the Crenarchaeota are not widespread, but are restricted to specialized habitats. Using sequence-based techniques that sidestep the cultivation of organisms, crenarchaeal small-subunit ribosomal RNA (rRNA) genes have been detected in open marine waters ${ }^{2,3}$. We now report the detection, in terrestrial lake and marsh sediments, of three novel, deeply divergent lineages of low-temperature Crenarchaeota, one of which includes the marine types. This finding indicates that such organisms are globally distributed and have an important role in the biosphere.

We isolated DNA directly from shallow-

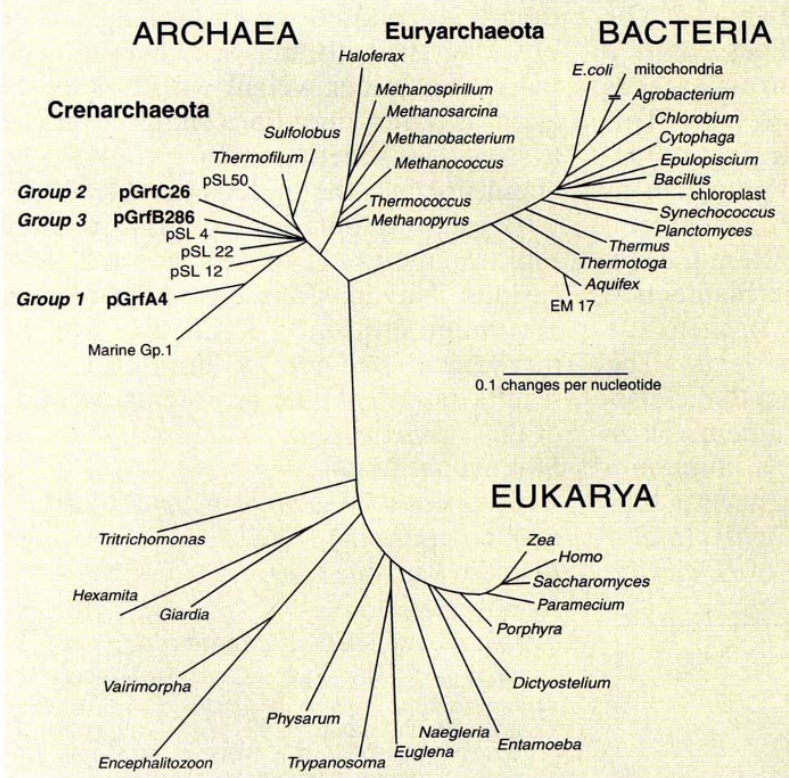

Phylogenetic analysis of terrestrial Crenarchaeota. DNA was purified directly from sediments collected from Lakes Griffy and Lemon, and used for PCR as outlined in the text and detailed previously ${ }^{4}$. Forward primers used were: 89F, 5'-GGCTCAGTAACGCGTAGTC-3'; 542F, 5'-CGCGGTAATACCAGCYC-3'; and 4Fa, 5'-TCCCGGTTGATCCTGCCRG-3'. All PCR products were generated using the universal reverse primer 1492RPL, 5'-GGCTCGAGCGGCCGCCCGGGTTACCTTGTTACGACTT-3'. PCR products were cloned as described, screened for unique types by restriction analysis and unique types were fully or partially sequenced. Sequences were aligned with others from the rRNA sequence database ${ }^{11}$ and phylogenetic analyses were done. The phylogenetic tree shown is a composite tree based on evolutionary distances ${ }^{12}$ and full sequences of representatives of the three clades discussed in the text. Clones pGrfA4, pGrfC26 and pGrfB286 represent groups 1, 2 and 3 Crenarchaeota, respectively. All sequences determined (31 partial and full sequences) have been deposited in the GenBank database, accession numbers U59968-U59999. Sequence alignments and bootstrap analyses of phylogenetic trees were as previously described ${ }^{4}$. Additional details of methods are available electronically (http://crab2.berkeley.edu/ pacelab/cren.html). a high-temperature ancestry for the lowtemperature organisms ${ }^{8,9}$. This correlation also suggests that the ability to grow at low temperatures has arisen within the Crenarchaeota in at least three separate instances.

The occurrence of diverse low-temperature Crenarchaeota in marine and terrestrial environments is unexpected, and shows that members of this kingdom of the phylogenetic domain Archaea are phenotypically more varied than was previously thought. Because neither these organisms nor their close, high-temperature relatives have been cultivated, we have no information about their metabolic properties. Considering the general metabolic capacities of other Archaea, however, we predict that the low-temperature Crenarchaeota can use molecular hydrogen as an energy source. The oxidation of hydrogen, generated abiotically and biotically, is a significant theme in microbial ecosystems ${ }^{10}$. The ubiquity of low-temperature Crenarchaeota in marine and terrestrial environments suggests that they are widely distributed in nature. The rRNA sequences provide tools for the study of their distribution and role in the global ecosystem.

\section{Karen L. Hershberger}

Department of Biology and Institute for Molecular and Cellular Biology,

Indiana University,

Bloomington, Indiana 47405, USA

\section{Susan M. Barns}

Department of Environmental Molecular Biology,

M888 Life Sciences Division,

Los Alamos National Laboratory,

Los Alamos, New Mexico 87545, USA

Anna-Louise Reysenbach

Department of Biochemistry and Microbiology,

Lipman Hall, Cook College,

Rutgers University,

New Brunswick, New Jersey 08903, USA

Scott C. Dawson

Norman R. Pace*

Department of Plant and Microbial Biology, Koshland Hall 111,

University of California, Berkeley,

California 94720-3102, USA

e-mail: nrpace@nature.berkeley.edu

\footnotetext{
1. Woese, C. R., Kandler, O. \& Wheelis, M. L. Proc. Natl Acad. Sci. USA 87, 4576-4579 (1990).

2. DeLong, E. F. Proc. Natl Acad. Sci. USA 89, 5685-5689 (1992).

3. Fuhrman, J. A., McCallum, K. \& Davis, A. A. Nature 356 148-149 (1992).

4. Barns, S. M., Fundyga, R. E., Jeffries, M. W. \& Pace, N. R. Proc. Natl Acad. Sci. USA 91, 1609-1613 (1994). 5. DeLong, E. F., Wu, K. Y., Prezelin, B. B. \& Jovine, R. V. M. Nature 371, 695-697 (1994).

6. Ueda, T., Suga, Y. \& Matsuguchi, T. Eur. J. Soil Sci. 46 415-421 (1995)

7. Barns, S. M., Delwiche, C. F., Palmer, J. D. \& Pace, N. R. Proc. Natl Acad. Sci. USA 93, 9188-9193 (1996).

8. Woese, C. R. Microbiol. Rev. 51, 221-271 (1987)

9. Pace, N. R. Cell 65, 531-533 (1991).

10. Stevens, T. O. \& McKinley, J. P. Science 270, 450-454 (1995).

11. Maidak, B. L. et al. Nucleic Acids Res. 24, 82-85

12. DeSoete, G. Psychometrika 48, 621-626 (1983).

* To whom correspondence should be addressed.
} 